\title{
Dose-rate-dependent damage of cerium dioxide in the scanning transmission electron microscope
}

\author{
Aaron C. Johnston-Peck ${ }^{a}$, Joseph S. DuChene ${ }^{b}$, Alan D. Roberts ${ }^{b}$, Wei David Wei ${ }^{b}$, and Andrew A. Herzing ${ }^{a}$ \\ ${ }^{a}$ Materials Measurement Lab, National Institute of Standards Technology, Gaithersburg, MD 20899 USA \\ ${ }^{b}$ Department of Chemistry and Center for Nanostructured Electronic Materials, University of Florida, \\ Gainesville, FL 32611 USA
}

\begin{abstract}
Beam damage caused by energetic electrons in the transmission electron microscope is a fundamental constraint limiting the collection of artifact-free information. Through understanding the influence of the electron beam, experimental routines may be adjusted to improve the data collection process. Investigations of $\mathrm{CeO}_{2}$ indicate that there is not a critical dose required for the accumulation of electron beam damage. Instead, measurements using annular dark field scanning transmission electron microscopy and electron energy loss spectroscopy demonstrate that the onset of measurable damage occurs when a critical dose rate is exceeded. The mechanism behind this phenomenon is that oxygen vacancies created by exposure to a $300 \mathrm{keV}$ electron beam are actively annihilated as the sample reoxidizes in the microscope environment. As a result, only when the rate of vacancy creation exceeds the recovery rate will beam damage begin to accumulate. This observation suggests that dose-intensive experiments can be accomplished without disrupting the native structure of the sample when executed using dose rates below the appropriate threshold. Furthermore, the presence of an encapsulating carbonaceous layer inhibits processes that cause beam damage, markedly increasing the dose rate threshold for the accumulation of damage.
\end{abstract}

\section{Keywords}

Scanning transmission electron microscopy

Point defects

Beam damage

Cerium dioxide

\section{Introduction}

In the transmission electron microscope damage to samples introduced through interactions with the high energy electron beam can limit measurement precision, resolution, and sensitivity. The processes that cause damage can be classified as being either elastic or inelastic in nature. A commonly encountered elastic damage process is ballistic or "knock-on" damage, which results from a direct transfer of momentum from the incident electrons to the nuclei of the atoms in the specimen. Radiolytic damage originates from inelastic interactions between the incident beam and electrons within the specimen, in which the potential energy of an excited atomic state is converted into atomic displacement. A more complete discussion on damage mechanisms has been reviewed elsewhere [1-4].

To limit the extent of such damage, the microscope operator can control a given set of experimental parameters, such as accelerating voltage, dose (electrons per unit area), and dose rate (dose per unit time) [5]. A critical dose defines the threshold above which a detectable amount of damage is produced within the sample. When the onset of damage occurs at a critical dose this means that low dose rates, short exposure times, or combinations thereof will limit damage. However, 
radiation-intense metrology, such as spectrum imaging and tomography, tests low-dose practices and emphasizes the need for understanding the mechanisms of electron beam damage and identifying techniques to mitigate such damage.

Cerium dioxide $\left(\mathrm{CeO}_{2}\right)$ has been the focus of several reports studying electron beam damage, which has been tracked using electron energy loss spectroscopy (EELS) to detect changes in the Ce $M_{4,5}$ edges [6-10]. The native oxidation state of the $\mathrm{Ce}$ ions in bulk $\mathrm{CeO}_{2}$ is +4 , although the presence of a reduced surface region has been commonly reported using scanning transmission electron microscopy (STEM)-EELS [8, 11-14]. As the electron beam interacts with the sample the Ce oxidation state will reduce to +3 . Results from dosimetry experiments have provided critical dose values for which a detectable change in the cerium oxidation state was observed [6-8]. This indicates that collecting artifact free data from $\mathrm{CeO}_{2}$ under dose intensive conditions would be problematic. However, recent studies by Turner et al. [11] and Goris et al. [12] hint the onset of beam damage in $\mathrm{CeO}_{2}$ may be dictated by some relationship other than a critical dose. Both studies involved high dose experimentation on $\mathrm{CeO}_{2}$ but, notably, also specifically reported that no measurable change in the sample was observed.

In an attempt to understand this apparent contradiction, a series of experiments were performed on $\mathrm{CeO}_{2}$ nanocubes. The nanocubes were exposed to a high energy electron beam using a series of dose rates while changes to the structure and chemistry of the nanocubes were monitored using low-angle and high-angle annular dark field STEM (LAADF- and HAADF-STEM, respectively) imaging and EELS measurements of the $\mathrm{Ce}_{4,5}$ edge. STEM imaging can be used to observe any changes to the structure such as the formation of voids or changes to the crystallographic phase. Additional information can be gained using annular dark field STEM imaging when the crystal is oriented (e.g., along a high-symmetry zone axis) to promote electron channeling. In this case, changes to the local crystal structure disrupt the electron channeling process, and alter the intensity in the resulting image. This technique has been used to identify strain fields around interfaces and line defects $[15,16]$. It is also sensitive to point defects $[17,18]$ and has been used to identify regions in $\mathrm{CeO}_{2}$ nanoparticles that were reduced [19]. By using this imaging technique in combination with EELS we were able to quantitatively assess the effects of electron beam irradiation in $\mathrm{CeO}_{2}$ nanocubes. We found dose rate to be the primary factor governing the reduction of $\mathrm{CeO}_{2}$ in the electron microscope. Below the critical dose rate threshold, no detectable damage accumulates. As a result, there is no well-defined critical dose, rather the dose necessary to observe a change in the oxidation state depends on the dose rate used for observation.

\section{Experimental Procedures}

$\mathrm{CeO}_{2}$ nanocubes were synthesized using wet chemical methods according to a previous literature report [20]. Briefly, $9.60 \mathrm{~g}$ sodium hydroxide $(\mathrm{NaOH})$ and $0.868 \mathrm{~g}$ of $\mathrm{Ce}\left(\mathrm{NO}_{3}\right)_{3}$ were dissolved in $50 \mathrm{~mL} \mathrm{H} \mathrm{H}_{2} \mathrm{O}$ (Nanopure $\mathrm{H}_{2} \mathrm{O}$ ) with a resistivity of approximately $18.2 \mathrm{M} \Omega \cdot \mathrm{cm}$ and sonicated for $10 \mathrm{~min}$. The solution was heated at $180^{\circ} \mathrm{C}$ for $24 \mathrm{~h}$ in a Teflon ${ }^{\circledR}$-lined autoclave. Afterwards, the samples were cooled to ambient temperature and washed with Nanopure $\mathrm{H}_{2} \mathrm{O}$. The solution was centrifuged three times at $837.8 \mathrm{rad} / \mathrm{s}$ for $5 \mathrm{~min}$ and dried in an oven at $60^{\circ} \mathrm{C}$ overnight to obtain a dry $\mathrm{CeO}_{2}$ powder. The particles were dispersed in water and deposited onto copper grids coated with carbon films and allowed to dry. The samples were cleaned using a low energy, inductively coupled plasma with a composition of $25 \% \mathrm{O}_{2}$ and $75 \% \mathrm{Ar}$. This was done to remove sources of hydrocarbon contamination and prevent the buildup of carbon as the sample was irradiated. The plasma treatments lasted $5 \mathrm{~s}$ to $20 \mathrm{~s}$ in length and were found to eliminate electron-beam-induced carbon deposition, but did not severely degrade the support film. Images taken before and after a plasma treatment indicate that no obvious restructuring of the nanocube occurred.

STEM data was collected using an aberration-corrected FEI Titan 80-300 operating at $300 \mathrm{kV}$. LAADF and HAADF images were acquired using the same detector by changing the camera length such 
that the inner collection semiangle was $\approx 28 \mathrm{mrad}$ and $\approx 88 \mathrm{mrad}$, respectively. The convergence semiangle used was $\approx 14$ mrad. EELS measurements were collected using an imaging energy filter (Gatan, Tridiem 865 ). The collection semiangle was $\approx 11 \mathrm{mrad}$ and a dispersion of $0.2 \mathrm{eV} / \mathrm{ch}$ was used. The FWHM of the zero loss peak was $\approx 1.0 \mathrm{eV}$ to $\approx 1.4 \mathrm{eV}$. To access the range of dose rates used in the experiments the gun lens focus was adjusted to change the probe current without interruption.

For all irradiation experiments, a 1024 x 1024 raster with a per-pixel dwell time of $1 \mu$ s was used. EELS spectra were recorded approximately every $30 \mathrm{~s}$ or $60 \mathrm{~s}$. A spectral acquisition time of $3 \mathrm{~s}$ was used, during which the electron beam was scanned over the entire square raster pattern encompassing the particle approximately three times; as a result, the measurement indicates the spatially-averaged oxidation state of the entire particle. Each image frame of the raster was recorded and stored except in experiments that lasted longer than $15 \mathrm{~min}$. In those cases, images were recorded and stored approximately every minute because of the limitations related to storing the large volume of data in temporary memory. The electron dose rate $\left(\mathrm{e} \cdot \mathrm{nm}^{-2} \cdot \mathrm{s}^{-1}\right)$ was calculated by dividing the probe current by the area of the raster. This is a time-averaged value, reflecting that each point of the specimen is not continuously illuminated, rather only momentarily once every complete raster. The cumulative dose $\left(\mathrm{e} \cdot \mathrm{nm}^{-2}\right)$ was calculated by multiplying the dose rate by the elapsed time. The probe currents were measured using the microscope's fluorescent screen that had been previously calibrated using a pico-ammeter.

The ratio of the integrated intensity of the $\mathrm{Ce} \mathrm{M}_{5}$ to the $\mathrm{Ce}_{4}$ peak - the "white-line" ratio was calculated by taking the second derivative of the spectra and integrating the positive portion of each edge. This procedure was implemented in Digital Micrograph using a publicly available script [21]. The uncertainty of these measurements is defined through the following relationship,

$$
\delta W=W \sqrt{\left(\frac{\sqrt{N_{1}}}{N_{1}}\right)^{2}+\left(\frac{\sqrt{N_{2}}}{N_{2}}\right)^{2}}
$$

where $W$ is the white-line ratio and $N_{1}$ and $N_{2}$ are the integrated intensities of the $C_{4,5} M_{4,5}$ edges.

\section{Results}

We monitored the changes to an individual $\mathrm{CeO}_{2}$ nanocube as a function of dose rate and cumulative electron dose. The nanocube (Figure 1), approximately (32 to 33) nm on a side, was oriented along the [001] zone axis. In Figure 2 the ratio of the integrated intensity of the $C_{2} M_{5}$ to the Ce $M_{4}$ peak $\left(\mathrm{M}_{5} / \mathrm{M}_{4}\right)$ - the white-line ratio, which is sensitive to oxidation state - is plotted as a function of dose. Each curve represents a different dose rate and all these data were obtained from the same nanocube. Several minutes elapsed between the start and stop of each experiment. Here we can observe that total dose does not correlate to changes in the $\mathrm{M}_{5} / \mathrm{M}_{4}$ ratio; rather dose rate is the key factor. At a dose rate of $2.97 \times 10^{5} \mathrm{e} \cdot \mathrm{nm}^{-2} \cdot \mathrm{s}^{-1}$ the nanocube was exposed to a dose of $9.03 \times 10^{8} \mathrm{e} \cdot \mathrm{nm}^{-2}$ after which no discernable change had occurred to the white-line ratio or to the fine structure of the $\mathrm{Ce}_{4,5}$ edges (Figure 2b). By comparison, when dose rates of $7.28 \times 10^{5} \mathrm{e} \cdot \mathrm{nm}^{-2} \cdot \mathrm{s}^{-1}, 1.10 \times 10^{6} \mathrm{e} \cdot \mathrm{nm}^{-2} \cdot \mathrm{s}^{-1}$, and $1.37 \times 10^{6}$ $\mathrm{e} \cdot \mathrm{nm}^{-2} \cdot \mathrm{s}^{-1}$ were used, an increase in the white-line ratio was observed, indicating the reduction of $\mathrm{Ce}$, even though the cumulative doses were lower. In addition, changes in the fine structure of the $\mathrm{Ce}_{4,5}$ edges could be observed at these dose rates (Figure $2 \mathrm{c}$ ). This includes a shift to lower energies, a reduction in the satellite peak intensities, and the change in the relative peak intensities, all of which are characteristic features of a transition from the +4 to +3 Ce oxidation state [6]. At the extreme of $1.37 \times$ $10^{6} \mathrm{e} \cdot \mathrm{nm}^{-2} \cdot \mathrm{s}^{-1}$, an almost immediate change to the white-lines ratio was observed. The initial data point had a white-lines ratio of $\approx 1.0$; this is larger than the average starting value $(\approx 0.94)$ of all the other measurements, suggesting that reduction of the nanocube had occurred almost immediately as it was exposed to the beam. After a dose of $4.12 \times 10^{7} \mathrm{e} \cdot \mathrm{nm}^{-2}$ the white-line ratio had a value of $\approx 1.05$ and 
increased to $\approx 1.14$ at a dose of $1.67 \times 10^{8} \mathrm{e} \cdot \mathrm{nm}^{-2}$. These doses are $\approx 4.6 \%$ and $\approx 18.5 \%$ of the total dose that caused no change to the white-line ratio when using a dose rate of $2.97 \times 10^{5} \mathrm{e} \cdot \mathrm{nm}^{-2} \cdot \mathrm{s}^{-1}$.

The HAADF-STEM images corroborate the behavior observed by EELS and a summary of all the results is outlined in Table 1 . In Figure 3, select images from each exposure are shown and the cumulative dose up to that point is indicated. Static displacements caused by the reduction disrupt electron channeling and reduce scatter to high angles. Additionally, the excitation of higher order Laue zones (HOLZ) will decrease with increasing lattice disorder. Therefore, the scatter to high angles decreases with increasing lattice disorder and the regions with reduced intensity will correspond to regions where reduction has taken place. Figure $3 a$ corresponds to the series acquired using a dose rate of $2.97 \times 10^{5} \mathrm{e} \cdot \mathrm{nm}^{-2} \cdot \mathrm{s}^{-1}$. It is evident that no marked changes to contrast occurred over the length of the exposure. By comparison, higher dose rates brought about significant change. In Figure $3 \mathrm{~b}$ (dose rate = $7.28 \times 10^{5} \mathrm{e} \cdot \mathrm{nm}^{-2} \cdot \mathrm{s}^{-1}$ ) the HAADF signal begins to decrease initially at exposed edges and propagates inwards leaving a bright core when the exposure was terminated. Under higher dose rates (Figure 3c, $1.10 \times 10^{6} \mathrm{e} \cdot \mathrm{nm}^{-2} \cdot \mathrm{s}^{-1}$ ) the contrast change spreads across the entire nanocube. At a still higher dose rate of $1.37 \times 10^{6} \mathrm{e} \cdot \mathrm{nm}^{-2} \cdot \mathrm{s}^{-1}$ (Figure $3 \mathrm{~d}$ ), an additional contrast transition was observed that was associated with vacancy ordering and the formation of $\mathrm{C}-\mathrm{Ce}_{2} \mathrm{O}_{3}$. Enlarged versions of the first and last frames can be seen in Figure 4, where the fast Fourier transform (FFT) inset of the final frame (Figure 4b) shows additional reflections corresponding to the partial conversion to $\mathrm{C}-\mathrm{Ce}_{2} \mathrm{O}_{3}$. Under all dose rate conditions, the nanocube retained its form, that is, the Ce sub-lattice remained relatively unchanged with the exception of slight changes in bond length that accompany the reduction process (often referred to as chemical expansion). No pores or protrusions formed, nor did any obvious bulk restructuring occur after a cumulative exposure of well over $1 \times 10^{9} \mathrm{e} \cdot \mathrm{nm}^{-2}$. A slight increase in the size of the $\{110\}$ facets was observed from $\approx 2 \mathrm{~nm}$ to $\approx 2.5 \mathrm{~nm}$. Additional nanocubes were subjected to similar experiments and displayed the same critical dose-rate behavior; a selection of those results can be found in the Supplemental Information.

To better understand this process another nanocube was studied. In this experiment, the nanocube was initially exposed to a low dose rate, then the dose rate was increased, and finally it was brought back down to the starting dose rate. The irradiation was continuous without interruptions, even during changes to the dose rate (probe current). Select frames are shown in Figure 5. In Figure 5a, the nanocube is being exposed to a low dose rate that does not cause damage to accumulate. Once the dose rate is significantly increased, damage is readily observed. The FFT (Figure $5 \mathrm{~d}$ ) shows the presence of new reflections (indicated by red arrows) and the FFT no longer matches with the [001] $\mathrm{CeO}_{2}$ simulated diffraction pattern (Figure 5f), but instead matches with that of [001] $\mathrm{C}-\mathrm{Ce}_{2} \mathrm{O}_{3}$ (Figure $5 e$ ). Additional data supporting the $\mathrm{C}-\mathrm{Ce}_{2} \mathrm{O}_{3}$ assignment is presented in the Supporting Information. The C$\mathrm{Ce}_{2} \mathrm{O}_{3}$ phase has a cubic superstructure with a lattice parameter approximately twice as large as the fluorite $\mathrm{CeO}_{2}$ structure. It is assigned to the la $\overline{3}$ space group with the oxygen vacancies preferentially localizing on the $16 c$ site $[22,23]$. However, this structure is metastable [24]; when the dose rate was returned to its original level and given several minutes to recover, the nanocube reverts back to its original state (Figure $5 c$ ). The FFT in Figure $5 c$ confirms that the nanocube has returned to the fluorite structure. This experiment demonstrates that the nanocube is first losing, and then acquiring, oxygen from the microscope environment (i.e., reversibly reducing and oxidizing). The ability of the particles to oxidize after being reduced was further corroborated using EELS. In a separate experiment a nanocube was initially exposed to a high-dose-rate condition and then the dose rate was reduced (Figure 6). As before, the irradiation was continuous without interruptions, even during changes to the dose rate (probe current). Under the high-dose-rate condition the $\mathrm{Ce}_{5} / \mathrm{M}_{4}$ ratio increases, indicating that reduction has occurred. Upon switching to the lower-dose condition, the $C e M_{5} / M_{4}$ ratio decreases, indicating oxidation. The reduction occurs when the dose rate is sufficiently high, while oxidation will occur even under irradiation as long as the dose rate is sufficiently low. 
To further explore this electron beam reduction of $\mathrm{CeO}_{2}$, a carbonaceous layer was deposited onto the same nanocube discussed in Figure 1,2 and 3. To deposit the carbonaceous layer the sample was removed from the microscope for approximately $120 \mathrm{~min}$ to expose it to the hydrocarbons present in ambient conditions. The sample was then placed back into the microscope and irradiated by the electron beam. The electron beam breaks the bonds of the organic species causing an amorphous layer of carbon to be deposited onto the nanocube [1]. Once the carbonaceous layer was deposited, the sample was plasma cleaned to remove the hydrocarbons and immediately loaded into the microscope. This final step was done to prevent further buildup of carbon when the nanocube was irradiated. An image of the carbon layer is shown in Figure 7a where a nonlinear (gamma) adjustment to the histogram was made to highlight the carbon layer in the LAADF-STEM image. After the carbon shell was deposited, the nanocube was exposed to an electron beam with a dose rate of $1.38 \times 10^{6} \mathrm{e} \cdot \mathrm{nm}^{-2} \cdot \mathrm{s}^{-1}$, the same dose rate that had previously reduced the nanocube and caused the formation of $\mathrm{C}-\mathrm{Ce}_{2} \mathrm{O}_{3}$. The EELS measurements (Figure 2) and HAADF-STEM images (Figure 3e) indicates that no reduction occurred. To confirm that no phase change had occurred, an FFT of the particle is shown (Supporting Information Figure S1) revealing that the structure is indeed fluorite $\mathrm{CeO}_{2}$. Figure $7 \mathrm{~b}$ (this image was also gamma adjusted) shows an image of the particle after this experiment, which reveals that the carbon layer did undergo some sputtering but remained intact throughout.

\section{Discussion}

The white-line ratio (Figure 2) and ADF-STEM image contrast (Figure 3) demonstrates that $\mathrm{CeO}_{2}$ nanoparticles can undergo significant reduction due to electron beam irradiation, this is in agreement with previous results $[6-9,13]$. When exposed to sufficiently high dose rates, long-range ordering of the oxygen vacancies occurs and $\mathrm{C}-\mathrm{Ce}_{2} \mathrm{O}_{3}$ forms (Figures 4 and 5). These observed behaviors do not depend on well-defined dose thresholds, rather we have systematically demonstrated it is the dose rate that is the critical factor to accumulate damage. This behavior originates because $\mathrm{CeO}_{2-x}$ can oxidize in the microscope environment and the electron-beam-stimulated changes are reversible (Figures 5 and 6). The reversibility of this process is in part supported by crystallography of the system. The fluorite structure of $\mathrm{CeO}_{2}$ is able to transport oxygen and support oxygen vacancies without irreversibly changing the $\mathrm{Ce}$ sub-lattice, even as $\mathrm{C}-\mathrm{Ce}_{2} \mathrm{O}_{3}$ forms [25]. As a result, the damage (i.e., oxygen vacancies) caused by the electron beam can be recovered as oxygen is absorbed from the vacuum environment. In this situation the accumulation of damage becomes a rate-determined process and it is only when the reduction (damage) rate exceeds the oxidation (recovery) rate that damage will accumulate.

The oxidation rate will depend on the adsorption of oxygen from the microscope environment and incorporation of it from the nanocube surface into the lattice. While oxidation of $\mathrm{CeO}_{2-x}$ or $\mathrm{C}-\mathrm{Ce}_{2} \mathrm{O}_{3}$ into $\mathrm{CeO}_{2}$ may seem counterintuitive given the sample environment is itself under a high vacuum (sample environment pressures on the order of $10^{-5} \mathrm{~Pa}$ to $10^{-6} \mathrm{~Pa}$ were used here), calculations demonstrate that the vacuum environment contains sufficient oxygen, or oxygen containing species (e.g., water can oxidize $\mathrm{CeO}_{2-x}[26]$ ) to oxidize the reduced nanocubes. The number of oxygen atoms present in the vacuum environment can be estimated using the ideal gas law. The pressure near the specimen is approximately $5 \times 10^{-5} \mathrm{~Pa}$; it is assumed that $10 \%$ is $\mathrm{O}_{2}$ (alternatively water vapor could be considered). The volume is assumed to be $0.01 \mathrm{~m}^{3}$ and the temperature was approximately $298 \mathrm{~K}$. This indicates that on the order of $10^{13} \mathrm{O}_{2}\left(\right.$ or $\mathrm{H}_{2} \mathrm{O}$ ) gas molecules would be present in the vacuum system. In a $30 \mathrm{~nm}$ cube of $\mathrm{CeO}_{2}$ there would be on the order of $10^{6}$ oxygen atoms, which means there is sufficient oxygen present in the vacuum to oxidize a sample that had been reduced. Examination of the Ellingham diagram indicates that $\mathrm{CeO}_{2}$ is the stable phase under these conditions. Accordingly, the kinetics of the 
oxidation process then becomes the pertinent question. The number of collisions that gas molecules will have with an object can be approximated by the following expression,

$$
Z=\frac{1}{4} \frac{N_{0} P}{R T} \sqrt{\frac{8 R T}{\pi M} A}
$$

where $N_{0}$ is the Avogadro constant, $P$ is pressure, $M$ is the molar mass of the gas species, and $A$ is the area of the surface. Assuming a $30 \mathrm{~nm}$ cube with one surface obscured indicates that $\approx 658 \mathrm{O}_{2}$ molecules would impinge on the surface per second. Assuming a sticking coefficient of 0.5 , this suggests that it would take approximately 10 minutes for enough oxygen to absorb on the surface to provide enough oxygen to support the oxidation a $30 \mathrm{~nm}$ cube from $\mathrm{Ce}_{2} \mathrm{O}_{3}$ to $\mathrm{CeO}_{2}$. The observed time required for a nanocube to oxidize from $\mathrm{Ce}_{2} \mathrm{O}_{3}$ to $\mathrm{CeO}_{2}$ was less (several minutes) but in reasonable agreement with the calculations. Differences between the calculations and observed behavior are likely due to assumptions (e.g., sticking coefficient or pressures) made in the calculations or spillover from the support.

The reduction process can be driven by either elastic or inelastic processes. The role of elastic processes is difficult to ascertain. The cross-sections for such events do not monotonically change with beam energy $[1,4]$ and values for the threshold energies for atomic sputtering and displacement are not available. By contrast, inelastic processes are expected to increase with decreasing beam energy $[1,5]$. This trend correlates well with the experimentally observed behavior, suggesting that inelastic processes play a more significant role in the beam-induced reduction of $\mathrm{CeO}_{2}$ nanoparticles. Several models have been proposed to describe beam-induced desorption from the region near the specimen surface [2729]. In an effort to better understand the reduction process at work in $\mathrm{CeO}_{2}$ nanoparticles, we have performed similar experiments using lower beam energies of $150 \mathrm{kV}$ and $80 \mathrm{kV}$ (see Supporting Information). In both cases, the overall behavior of the nanoparticles during beam exposure was similar to that which was observed at $300 \mathrm{kV}$. However, the critical dose rates necessary to reduce the nanocubes were found to decrease along with energy. This indicates that the cross sections for events that result in oxygen removal are inversely proportional to accelerating voltage.

One possible explanation for this behavior was suggested previously in a model that predicted a dose rate dependence similar to that which we have found [4, 30, 31]. In this model, beam damage arises due to charging and the resulting development of an electric field within the specimen. The incident electron beam stimulates the emission of secondary and Auger electrons from the exposed area, resulting in an electric field directed away from the irradiated region. The resulting charge imbalance is then offset through the transport of cations and anions, which travel away from and towards the irradiated area, respectively. As we have seen, in the case of $\mathrm{CeO}_{2}$ this transport is dominated by the highly mobile oxygen ions traveling away from the irradiated region (i.e. in the direction of the induced electric field). If the model is correct, this suggests that the oxygen ions become neutral or positively charged, perhaps through an Auger-related process [30], and then migrate along the electric field to desorb from the nanocube surface.

This model may also explain the observation that increasing the dose rate beyond a critical threshold does not result in further change in the white-line ratio (see Supporting Information Figure S6). This demonstrates that a steady-state condition is eventually reached and that the mechanism responsible for reducing the particle from $\mathrm{CeO}_{2}$ to $\mathrm{C}-\mathrm{Ce}_{2} \mathrm{O}_{3}$ cannot provide the additional driving force necessary to fully reduce the particle to the metallic state. The specimen charging model predicts that the surface potential would increase, which may suppress secondary electron emission. This would limit the flux of electrons away from the irradiated region, as well as the driving force to transport charge by the motion of ions. However, at present we cannot rule out other mechanisms which could explain the 
self-limiting nature of the reduction process. For example, an increase in the $\mathrm{Ce}-\mathrm{O}$ bonding energy from $\mathrm{CeO}_{2}$ to $\mathrm{C}-\mathrm{Ce}_{2} \mathrm{O}_{3}$ could also stabilize the particles against further reduction. Thermal decomposition may also play a role, but experimentation (see the Supporting Information for details) suggests this is not the primary mechanism driving the reduction process.

In short, we can identify the response of the system under irradiation, but we do not have a complete understanding of underlying physical mechanisms that are responsible for the beam damage. Nevertheless, identifying and understanding the implications of critical dose-rate behavior is important for practical experimentation. This suggests that dose intensive experiments, like those previously mentioned $[11,12]$, can be executed without altering the particle and generating artifacts in the data. Restricting artifacts can be managed by proper control of the probe current and other variables that influence reduction. If decreasing the dose rate unacceptably degrades signal to noise levels, multiple measurements could be recorded and averaged in post-processing to achieve the desired results. The use of environmental TEM (ETEM) techniques should increase the critical dose rate by increasing the frequency at which an oxygen molecule would interact with the $\mathrm{CeO}_{2}$. Alternatively, if an ultra-high vacuum system (sample environment pressures $<10^{-7} \mathrm{~Pa}$ ) were used this critical dose-rate behavior may be suppressed due to the slow delivery of oxidizing gaseous species to the particle surface. In cases where the anion is an element other than oxygen, it is possible that electron irradiation would displace the native anion and oxygen would then diffuse into the lattice to increase the oxygen content of the material. There is some evidence for this mechanism in $\mathrm{CeO}_{2}$ particles with fluorine impurities [8].

Lastly, the observed behavior of the nanocubes was strongly influenced by the presence of a carbon layer, which acted to raise the dose-rate threshold substantially. This observation is not without precedent; it has been reported that carbon coatings ameliorate beam-induced damage in metals [32], organics [33,34], and oxides [35, 36]. Here it is believed that the carbonaceous layer is a physical barrier that inhibits facile desorption of the oxygen atoms. Oxygen atoms which are displaced by electron beam interactions are able to recombine into the $\mathrm{CeO}_{2}$ lattice rather than enter the surrounding vacuum environment. As a result, the material does not reduce under the electron beam. The coating could also increase thermal and charge transport; if heating or charging of the sample assisted the reduction process then the carbon shell may also stymie those mechanisms. The carbon shell is only a temporary means of preventing beam damage, as it too is sputtered by the electron beam. Once its structural integrity is corrupted, the oxygen atoms would again be able to transfer into the surrounding atmosphere and the $\mathrm{CeO}_{2}$ would begin to reduce. The presence of a thin amorphous shell does not have a significant negative effect on the imaging process. It will increase the background signal but spatial resolution should remain relatively unaffected $[37,38]$. In certain cases, coating samples to eliminate damage may be a suitable approach. The electron-beam-mediated method used to coat the sample here is not suitable for large areas, nor is it the most practical. Techniques such as atomic layer deposition (ALD) can be used to controllably coat specimens, including nanoparticles, with thin amorphous layers [39]. Such a coating would not be limited solely to carbon, as other low atomicnumber materials, such as $\mathrm{Al}_{2} \mathrm{O}_{3}$, could also be considered.

\section{Conclusions}

Electron beam damage in $\mathrm{CeO}_{2}$ was tracked using EELS and STEM imaging. It was found that the dose rate itself was the critical factor determining whether damage, in the form of oxygen vacancies, accumulated in the sample. Below the dose-rate threshold damage does not accumulate. Above the dose rate threshold, oxygen vacancies form and the cerium ions reduced from the +4 to +3 oxidation state. If the dose rate is sufficiently high, long-range ordering of the oxygen vacancies transforms the phase of the particle from fluorite $\mathrm{CeO}_{2}$ to sesquioxide $\mathrm{C}_{-} \mathrm{Ce}_{2} \mathrm{O}_{3}$. Oxygen present in the microscope environment oxidizes the sample and recovers the electron-beam damage making this process 
reversible. Therefore, damage only accumulates when the recovery rate is slower than the damage rate. This dynamic relationship with the surrounding environment ensures that dose-heavy experiments are tractable if appropriate steps are taken to keep the dose rate low. Diffusion barriers, such as an amorphous coating, make the nanocubes more resistant to beam damage and can been utilized to facilitate high-dose-rate measurements.

\section{Acknowledgements}

This research was performed while A.C.J.-P. held a National Research Council Research Associateship Award at the National Institute of Standards and Technology. W.D.W., J.S.D., and A.D.R. thank the Air Force Office of Scientific Research under AFOSR Award No. FA9550-14-1-0304 and the National Science Foundation for support under Grant CHE-1308644 and the CCI Center for Nanostructured Electronic Materials (CHE-1038015). A.D.R. acknowledges the University of Florida Howard Hughes Medical Institute Intramural Award.

\section{Figures}

Table 1. A summary of the HAADF-STEM and EELS measurements as a function of dose rate for a single nanocube. The particle filled $\approx 80 \%$ of the square raster.

\begin{tabular}{lcllll}
\hline $\begin{array}{l}\text { Dose Rate } \\
\left(\mathrm{e} \cdot \mathrm{nm}^{-2} \cdot \mathrm{s}^{-1}\right)\end{array}$ & $\begin{array}{l}\text { Probe Curent } \\
(\mathrm{pA})\end{array}$ & $\begin{array}{l}\text { Cumulative } \\
\text { Dose }\left(\mathrm{e} \cdot \mathrm{nm}^{-2}\right)\end{array}$ & EELS & $\begin{array}{l}\text { HAADF Contrast } \\
\text { Change }\end{array}$ & Phase Change \\
\hline $2.97 \times 10^{5}$ & 65 & $9.03 \times 10^{8}$ & No Change & None & None \\
$7.28 \times 10^{5}$ & 159 & $5.23 \times 10^{8}$ & Reduction & Yes & None \\
$1.10 \times 10^{6}$ & 240 & $4.28 \times 10^{8}$ & Reduction & Yes & None \\
$1.37 \times 10^{6}$ & 300 & $6.60 \times 10^{8}$ & Reduction & Yes & $\mathrm{CeO}_{2}$ to $\mathrm{C}^{-\mathrm{Ce}_{2} \mathrm{O}_{3}}$ \\
$1.38 \times 10^{6}+$ C Shell & 301 & $7.45 \times 10^{8}$ & No Change & None & None \\
\hline
\end{tabular}

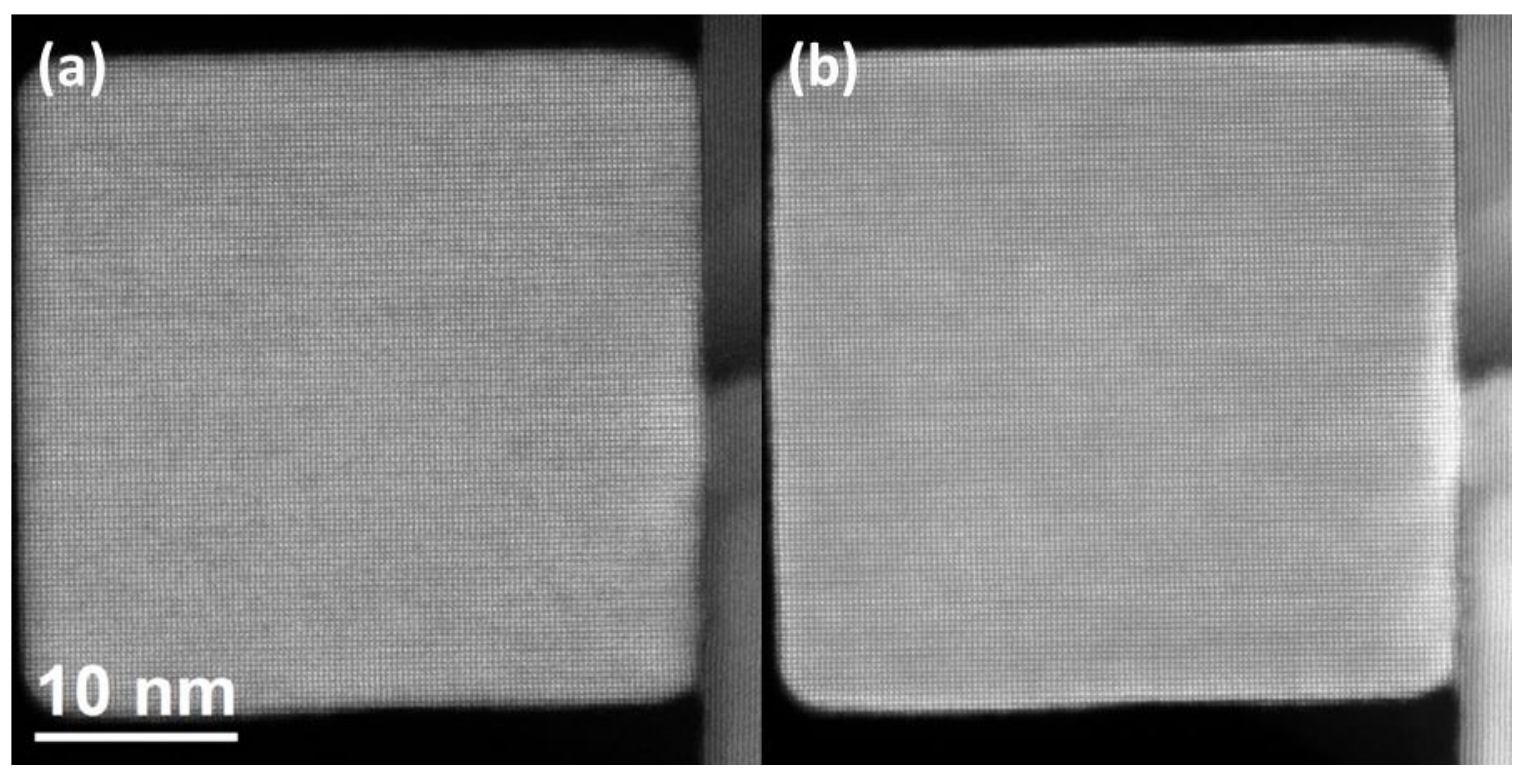

Figure 1. LAADF-STEM (a) and HAADF-STEM (b) images of a $\mathrm{CeO}_{2}$ nanocube orientated along the [001] direction. Differences in contrast between the two images at the nanocube edge are due to atomic static displacements. 

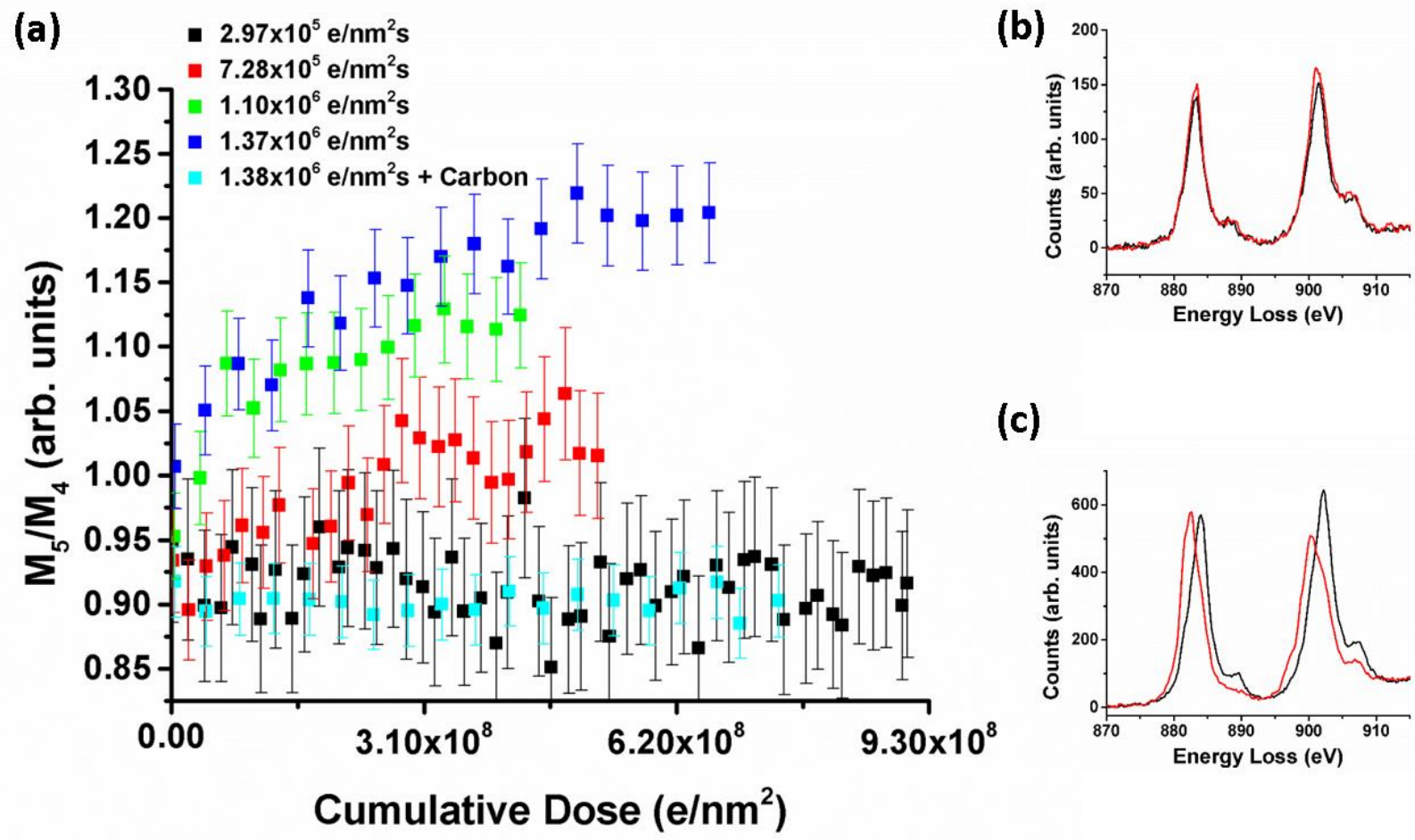

(c)

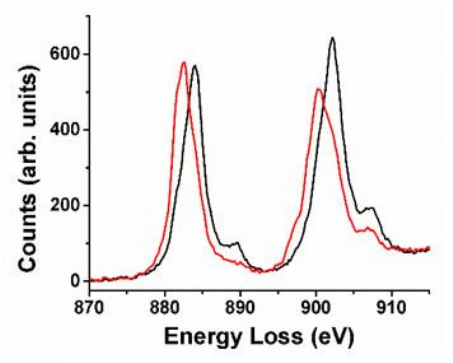

Figure 2. The white-line ratio of the $\mathrm{Ce}_{4,5}$ edges from a single nanocube as a function of cumulative dose under different dose rates (a). The $\mathrm{Ce}_{4,5}$ edges $(b, c)$ at the initial (black curve) and final state (red curve) of the exposure experiments under two different dose rates, (b) $2.97 \times 10^{5} \mathrm{e} \cdot \mathrm{nm}^{-2} \cdot \mathrm{s}^{-1}$ and (c) 1.37 $\times 10^{6} \mathrm{e} \cdot \mathrm{nm}^{-2} \cdot \mathrm{s}^{-1}$. 


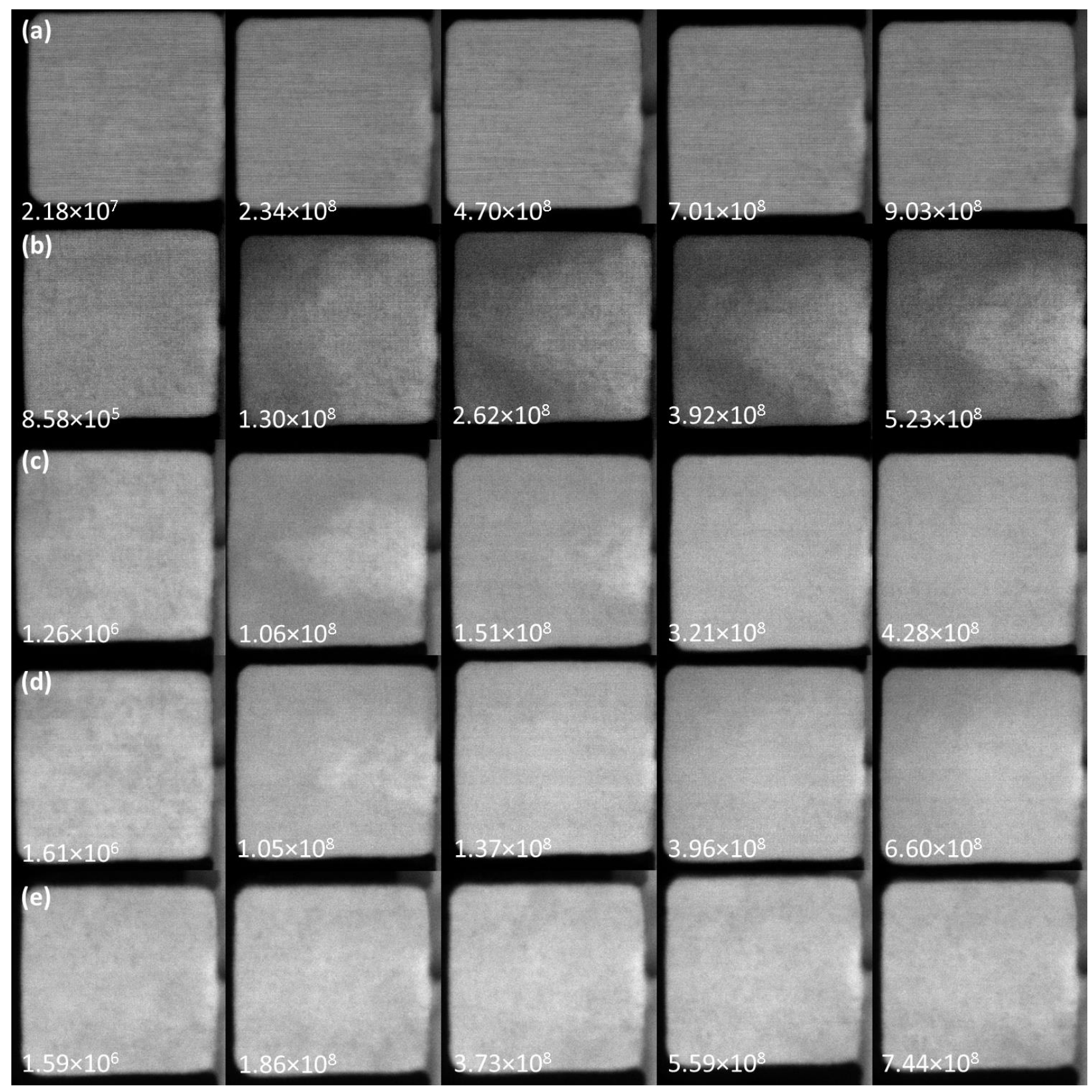

Figure 3. HAADF-STEM images of $\mathrm{a} \mathrm{CeO}_{2}$ nanocube as a function of dose rate $\left(\mathrm{a}-2.97 \times 10^{5} \mathrm{e} \cdot \mathrm{nm}^{-2} \cdot \mathrm{s}^{-1}, \mathrm{~b}\right.$ $-7.28 \times 10^{5} \mathrm{e} \cdot \mathrm{nm}^{-2} \cdot \mathrm{s}^{-1}, \mathrm{c}-1.10 \times 10^{6} \mathrm{e} \cdot \mathrm{nm}^{-2} \cdot \mathrm{s}^{-1}, \mathrm{~d}-1.37 \times 10^{6} \mathrm{e} \cdot \mathrm{nm}^{-2} \cdot \mathrm{s}^{-1}, \mathrm{e}-1.38 \times 10^{6} \mathrm{e} \cdot \mathrm{nm}^{-2} \cdot \mathrm{s}^{-1}$ ) and total cumulative dose (from left to right in a given row). The cumulative dose (units of $\mathrm{e} \cdot \mathrm{nm}^{-2}$ ) over time is indicated in the lower left corner. No contrast change is apparent under low-dose-rate conditions (a) or when a carbonaceous shell covers the nanocube (e). 


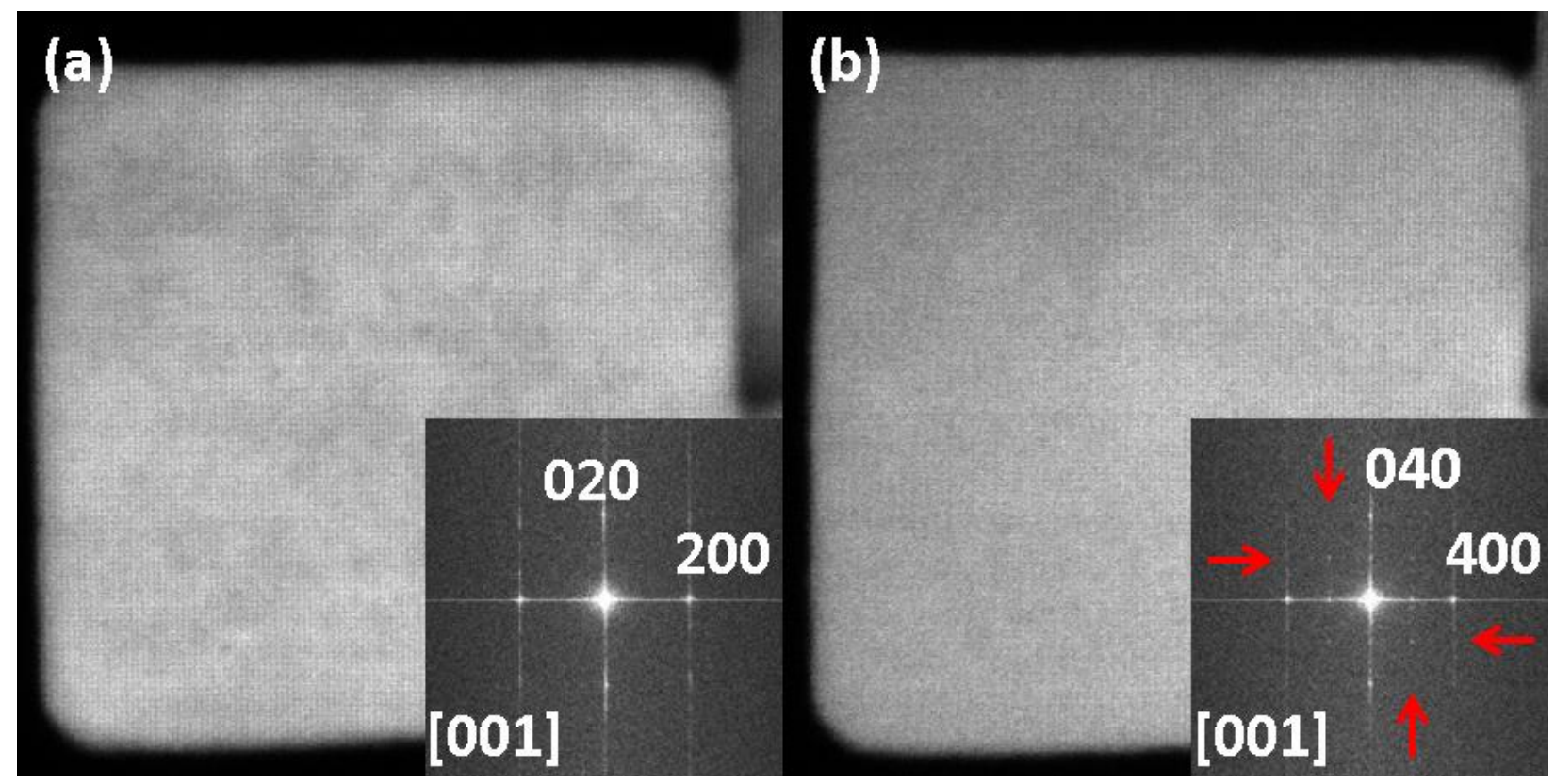

Figure 4. HAADF-STEM images of a nanocube in its original fluorite $\mathrm{CeO}_{2}$ structure (a) and after being converted to the sesquioxide $\mathrm{C}-\mathrm{Ce}_{2} \mathrm{O}_{3}$ phase (b). The red arrows in the FFT point to additional reflections that appear upon transition to the sesquioxide phase.

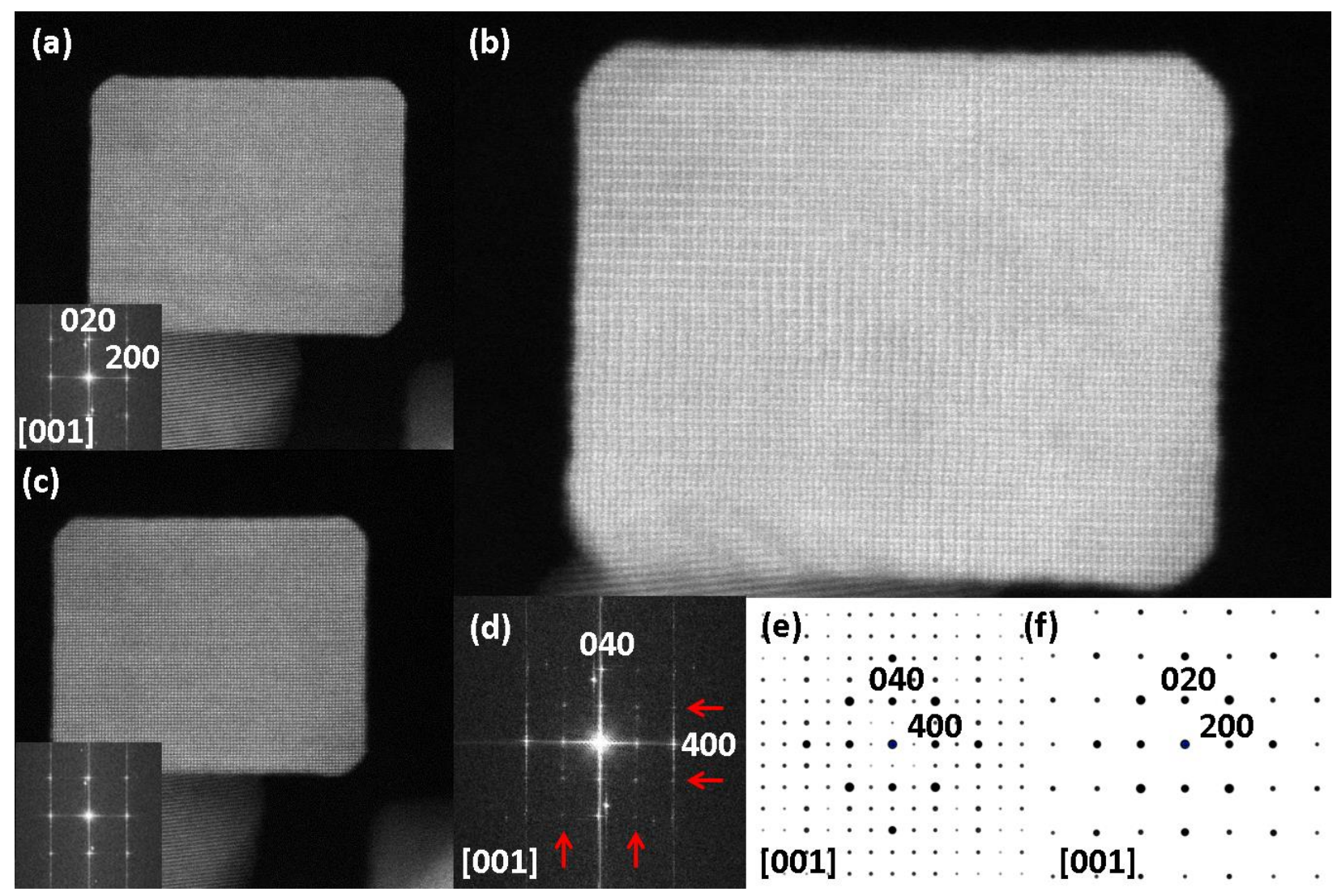

Figure 5. LAADF-STEM images of a [001] oriented $\mathrm{CeO}_{2}$ nanocube with edge-lengths of $16 \mathrm{~nm}$ and 21 $\mathrm{nm}$. Under intense irradiation it transforms from its original fluorite structure (a) to the sesquioxide C- 
$\mathrm{Ce}_{2} \mathrm{O}_{3}$ phase (b) and back to its original form (c) when the dose rate is reduced. The FFT (d) is generated from (b). Additional reflections indicated by red arrows can be observed in comparison to the FFT shown in (a) and (c). Simulated diffraction patterns from the $\mathrm{C}-\mathrm{Ce}_{2} \mathrm{O}_{3}(e)$ and $\mathrm{CeO}_{2}$ (f) illustrate differences between the structures.

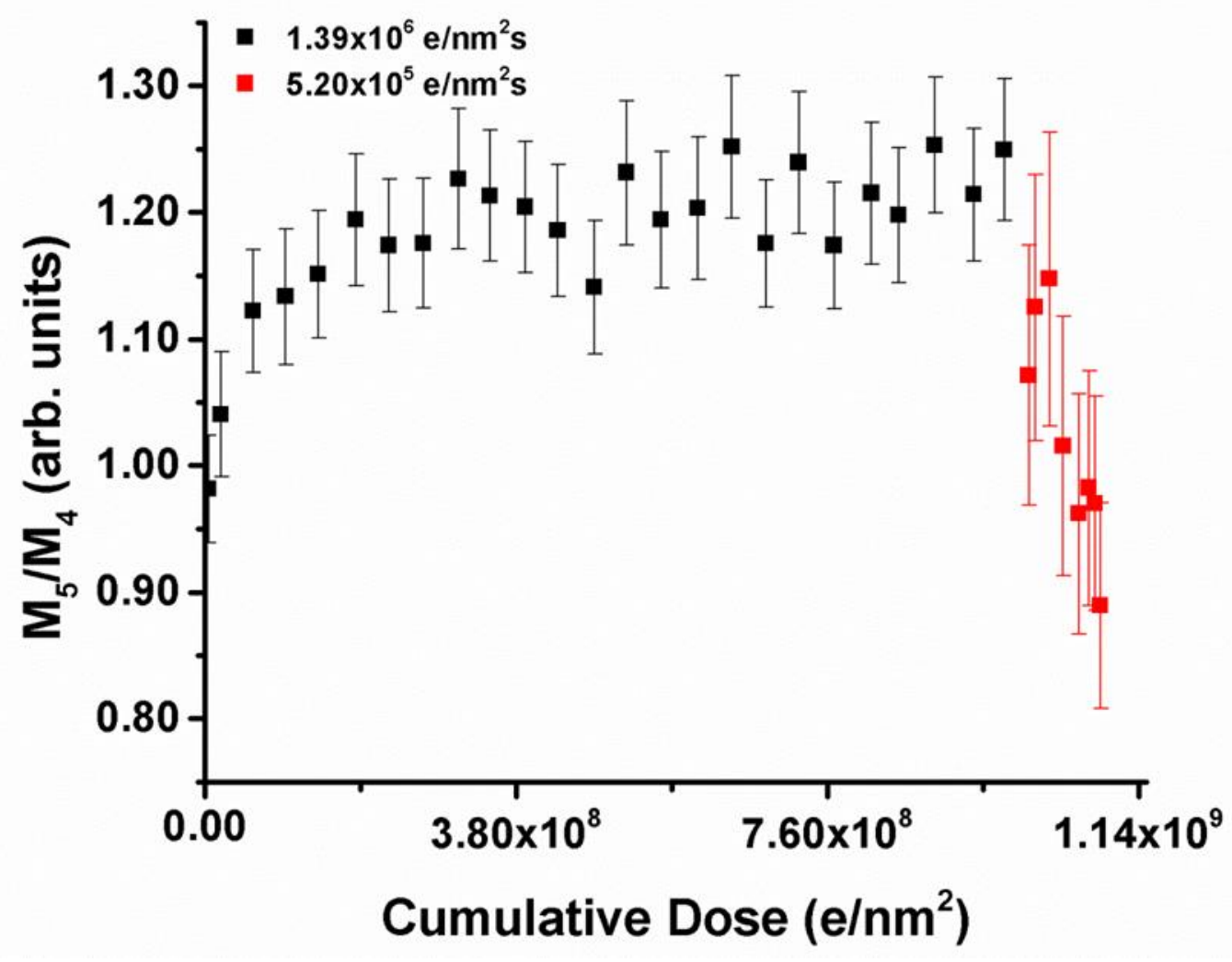

Figure 6. The white-line ratio of $\mathrm{Ce}_{4,5}$ edges as a function of cumulative dose under a high dose rate (black) followed by a lower dose rate (red). The nanocube reduces upon exposure to a high dose rate and oxidizes upon transitioning to a lower dose rate. 

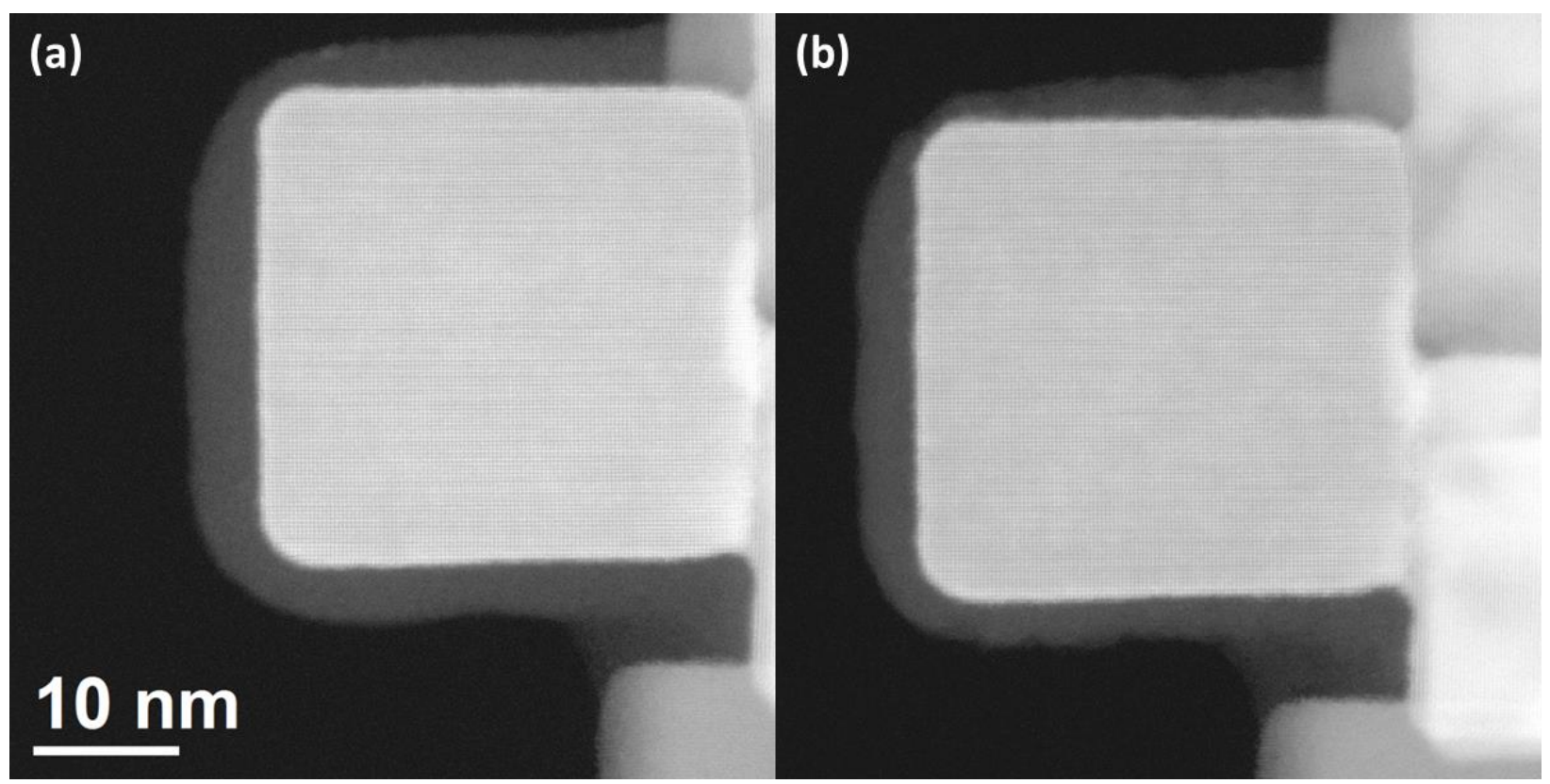

Figure 7. LAADF-STEM images of a $\mathrm{CeO}_{2}$ nanocube with the as-deposited carbonaceous shell (a) and after it was exposed to a high-flux electron beam (b). The images were gamma adjusted to highlight the carbonaceous shell.

\section{References}

[1] R.F. Egerton, P. Li, M. Malac, Radiation damage in the TEM and SEM, Micron, 35 (2004) 399-409.

[2] R.F. Egerton, R. McLeod, F. Wang, M. Malac, Basic questions related to electron-induced sputtering in the TEM, Ultramicroscopy, 110 (2010) 991-997.

[3] L.W. Hobbs, F.W. Clinard Jr, S.J. Zinkle, R.C. Ewing, Radiation effects in ceramics, Journal of Nuclear Materials, 216 (1994) 291-321.

[4] N. Jiang, Electron beam damage in oxides: A review, Reports on Progress in Physics, 79 (2016) 016501.

[5] R.F. Egerton, Control of radiation damage in the TEM, Ultramicroscopy, 127 (2013) 100-108.

[6] L.A.J. Garvie, P.R. Buseck, Determination of $\mathrm{Ce}^{4+} / \mathrm{Ce}^{3+}$ in electron-beam-damaged $\mathrm{CeO}_{2}$ by electron energy-loss spectroscopy, Journal of Physics and Chemistry of Solids, 60 (1999) 1943-1947.

[7] R. Sharma, P.A. Crozier, Z.C. Kang, L. Eyring, Observation of dynamic nanostructural and nanochemical changes in ceria-based catalysts during in-situ reduction, Philosophical Magazine, 84 (2007) 2731-2747.

[8] S.R. Gilliss, J. Bentley, C.B. Carter, Electron energy-loss spectroscopic study of the surface of ceria abrasives, Applied Surface Science, 241 (2005) 61-67.

[9] J. Bentley, S.R. Gilliss, C.B. Carter, J.F. Al-Sharab, F. Cosandey, I.M. Anderson, P.J. Kotula, Nanoscale EELS analysis of oxides: composition mapping, valence determination and beam damage, Journal of Physics: Conference Series, 26 (2006) 69.

[10] J.P. Winterstein, C.B. Carter, Electron-beam damage and point defects near grain boundaries in cerium oxide, Journal of the European Ceramic Society, 34 (2014) 3007-3018.

[11] S. Turner, S. Lazar, B. Freitag, R. Egoavil, J. Verbeeck, S. Put, Y. Strauven, G. Van Tendeloo, High resolution mapping of surface reduction in ceria nanoparticles, Nanoscale, 3 (2011) 3385-3390.

[12] B. Goris, S. Turner, S. Bals, G. Van Tendeloo, Three-dimensional valency mapping in ceria nanocrystals, ACS Nano, 8 (2014) 10878-10884. 
[13] S.J. Haigh, N.P. Young, H. Sawada, K. Takayanagi, A.I. Kirkland, Imaging the active surfaces of cerium dioxide nanoparticles, ChemPhysChem, 12 (2011) 2397-2399.

[14] L. Wu, H.J. Wiesmann, A.R. Moodenbaugh, R.F. Klie, Y. Zhu, D.O. Welch, M. Suenaga, Oxidation state and lattice expansion of $\mathrm{CeO}_{2-x}$ nanoparticles as a function of particle size, Physical Review B, 69 (2004) 125415.

[15] Z. Yu, D.A. Muller, J. Silcox, Study of strain fields at a-Si/c-Si interface, Journal of Applied Physics, 95 (2004) 3362-3371.

[16] P.J. Phillips, M. De Graef, L. Kovarik, A. Agrawal, W. Windl, M.J. Mills, Atomic-resolution defect contrast in low angle annular dark-field STEM, Ultramicroscopy, 116 (2012) 47-55.

[17] D.A. Muller, N. Nakagawa, A. Ohtomo, J.L. Grazul, H.Y. Hwang, Atomic-scale imaging of nanoengineered oxygen vacancy profiles in $\mathrm{SrTiO}_{3}$, Nature, 430 (2004) 657-661.

[18] D.D. Perovic, C.J. Rossouw, A. Howie, Imaging elastic strains in high-angle annular dark field scanning transmission electron microscopy, Ultramicroscopy, 52 (1993) 353-359.

[19] A.C. Johnston-Peck, J.P. Winterstein, A.D. Roberts, J.S. DuChene, K. Qian, B.C. Sweeny, W.D. Wei, R. Sharma, E.A. Stach, A.A. Herzing, Oxidation-state sensitive imaging of cerium dioxide by atomicresolution low-angle annular dark field scanning transmission electron microscopy, Ultramicroscopy, 162 (2016) 52-60.

[20] L. Torrente-Murciano, A. Gilbank, B. Puertolas, T. Garcia, B. Solsona, D. Chadwick, Shapedependency activity of nanostructured $\mathrm{CeO}_{2}$ in the total oxidation of polycyclic aromatic hydrocarbons, Applied Catalysis B: Environmental, 132-133 (2013) 116-122.

[21] D.R.G. Mitchell, B. Schaffer, Scripting-customised microscopy tools for Digital Micrograph ${ }^{\mathrm{TM}}$, Ultramicroscopy, 103 (2005) 319-332.

[22] N. Hirosaki, S. Ogata, C. Kocer, Ab initio calculation of the crystal structure of the lanthanide $\operatorname{Ln}_{2} \mathrm{O}_{3}$ sesquioxides, Journal of Alloys and Compounds, 351 (2003) 31-34.

[23] E.A. Kümmerle, G. Heger, The Structures of $\mathrm{C}-\mathrm{Ce}_{2} \mathrm{O}_{3+\delta}, \mathrm{Ce}_{7} \mathrm{O}_{12}$, and $\mathrm{Ce}_{11} \mathrm{O}_{20}$, J. Solid State Chem., 147 (1999) 485-500.

[24] L. Petit, A. Svane, Z. Szotek, W.M. Temmerman, First-principles study of rare-earth oxides, Physical Review B, 72 (2005) 205118.

[25] Z.L. Wang, Z.C. Kang, Functional and smart materials: Structural evolution and structure analysis, Plenum Press, 1998.

[26] D.R. Mullins, P.M. Albrecht, T.-L. Chen, F.C. Calaza, M.D. Biegalski, H.M. Christen, S.H. Overbury, Water dissociation on $\mathrm{CeO}_{2}(100)$ and $\mathrm{CeO}_{2}(111)$ thin films, The Journal of Physical Chemistry C, 116 (2012) 19419-19428.

[27] M.L. Knotek, P.J. Feibelman, Ion desorption by core-hole auger decay, Physical Review Letters, 40 (1978) 964-967.

[28] P.R. Antoniewicz, Model for electron- and photon-stimulated desorption, Physical Review B, 21 (1980) 3811-3815.

[29] D. Menzel, R. Gomer, Desorption from metal surfaces by low-energy electrons, The Journal of Chemical Physics, 41 (1964) 3311-3328.

[30] J. Cazaux, Correlations between ionization radiation damage and charging effects in transmission electron microscopy, Ultramicroscopy, 60 (1995) 411-425.

[31] N. Jiang, Damage mechanisms in electron microscopy of insulating materials, Journal of Physics D: Applied Physics, 46 (2013) 305502.

[32] D.A. Muller, J. Silcox, Radiation damage of $\mathrm{Ni}_{3} \mathrm{Al}$ by 100 keV electrons, Philosophical Magazine A, 71 (1995) 1375-1387.

[33] J.R. Fryer, Radiation damage in organic crystalline films, Ultramicroscopy, 14 (1984) 227-236.

[34] J.R. Fryer, F. Holland, The reduction of radiation damage in the electron microscope, Ultramicroscopy, 11 (1983) 67-70. 
[35] M.I. Buckett, L.D. Marks, Electron irradiation damage in NiO, Surface Science, 232 (1990) 353-366. [36] J. Strane, L.D. Marks, D.E. Luzzi, M.I. Buckett, J.P. Zhang, B.W. Wessels, Encapsulation, diffusion and DIET in the electron microscope, Ultramicroscopy, 25 (1988) 253-257.

[37] N. de Jonge, N. Poirier-Demers, H. Demers, D.B. Peckys, D. Drouin, Nanometer-resolution electron microscopy through micrometers-thick water layers, Ultramicroscopy, 110 (2010) 1114-1119.

[38] K.A. Mkhoyan, S.E. Maccagnano-Zacher, E.J. Kirkland, J. Silcox, Effects of amorphous layers on ADFSTEM imaging, Ultramicroscopy, 108 (2008) 791-803.

[39] H. Feng, J. Lu, P. Stair, J. Elam, Alumina over-coating on Pd nanoparticle catalysts by atomic layer deposition: Enhanced stability and reactivity, Catal Lett, 141 (2011) 512-517. 